\title{
Heart failure and central sleep apnea in the era of implantable recorders
}

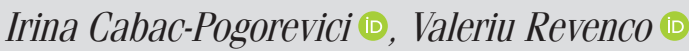

Department of Cardiology, Nicolae Testemitanu State University of Medicine and Pharmacy; Chisinau-Republic of Moldova

\section{ABSTRACT}

Sleep-disordered breathing (SDB) is a complex syndrome with a high prevalence and a significant impact on the general well-being of the overall population. Heart failure (HF) is a major health issue with an increasing incidence, a high rate of hospitalizations, and high mortality in developing countries. Focusing on early recognition and management of HF comorbidities may have an important role in reducing the economic burden and public health impact of HF. The close interconnection between HF, heart rhythm disturbances, and sleep apnea is supported by the mutual risk factors such as age, smoking, obesity, and male sex. Central sleep apnea (CSA) may be considered a marker of advanced HF, often being associated with elevated pulmonary capillary wedge pressure, brain natriuretic peptide (BNP), and noradrenaline levels and with low left ventricular ejection fraction. In the same way, there is an important correlation between CSA and different types of arrhythmias. The large intraindividual rhythm variability reported in patients with SDB who underwent continuous monitoring by implantable loop recorder (ILR) demonstrated the incapacity of 24-hour and 48-hour Holter monitoring to accurately determine the incidence of cardiac arrhythmias. In patients with HF and CSA, the extended cardiac monitoring by ILR becomes compulsory because in-time interventions could be life saving, but with the absolute lack of solid evidence in this field, there is an acute need for extensive randomized trials to further highlight the potential beneficial effect of ILR monitoring in patients with CSA and HF.

Key words: heart failure, central sleep apnea, cardiac arrhythmias

Cite this article as: Cabac-Pogorevici I, Revenco V. Heart failure and central sleep apnea in the era of implantable recorders. Anatol J Cardiol 2021; 25: 216-24.

\section{Introduction}

Sleep-disordered breathing (SDB) is a complex syndrome with a high prevalence and a significant impact on the general wellbeing of the overall population. Sleep is a basic vital process of the human body, being thoroughly controlled by the human brain. The sleeping phases alternate between rapid eye movement (REM) and non-REM. This process is the result of the continuous interaction between the sympathetic and parasympathetic nervous system with the dominance of the vagal tone. This complex interaction allows the relaxation of the cardiovascular-neural axis with further rest and restoration of the balance in the human body, resulting in a normal activity of the cardiovascular, nervous, and endocrine systems in the vigil period. Sudden changes in the balance of the vegetative nervous system cause the disturbance of the interaction and alternation of the sleeping phases; thus, all the types of sleep disturbances, especially sleep apnea, have an important negative impact on the nervous, endocrine, and especially the cardiovascular system.
SDB includes a wide range of pathologies, which can be generally classified into several subtypes, such as obstructive sleep apnea (OSA) [daytime sleepiness with at least five obstructive events per hour (apneas+hypopneas)], central sleep apnea (CSA) (daytime sleepiness or frequent arousals with at least five central events per hour), and sleep-related hypoventilation (1).

SDB is frequently associated with heart failure (HF) with a prevalence of about $40 \%-50 \%$ in these patients (2). OSA as well as CSA are often found in patients with HF, although OSA is being considered as a well-known cause of HF, whereas CSA is rated as a consequence of it (3). SDB is widely recognized as an important contributor to HF-associated morbidity and mortality. So, considering the high prevalence of HF and HF-associated comorbidities in the last decades, an important attention has been drawn to the understanding of the pathophysiological mechanisms, early detection methods, and management strategies of SDB, especially of the different types of sleep apnea (4).

The early recognition and treatment of sleep apnea are important for the improvement of quality of life $(0 \mathrm{oL})$ and prevention of the earlier-mentioned consequences. The diagnosis of

Address for Correspondence: Irina Cabac-Pogorevici, MD, Department of Cardiology, Nicolae Testemitanu State University of Medicine and Pharmacy; Chisinau-Republic of Moldova Phone: +37369154544 E-mail: irinutzai@yahoo.com

Accepted Date: 01.02.2021 Available Online Date: 16.03.2021

(C) Copyright 2021 by Turkish Society of Cardiology - Available online at www.anatoljcardiol.com DOI:10.5152/AnatolJCardiol.2021.63668 


\section{HIGHLIGHTS}

- The arrhythmic implications of central sleep apnea (CSA) in patients with heart failure (HF) remain controversial.

- The new monitoring strategies may switch the use of implantable monitors from arrhythmia detection to a heart disease management strategy.

- There is no certainty about the therapeutic approach for the treatment of CSA in patients with HF; further research is required for the establishment of strong evidence for the new or conventional treatment options before applying them to clinical practice.

- The elevated rate of asymptomatic high-risk arrhythmias in patients with HF and CSA makes extended cardiac monitoring by implantable loop recorder compulsory in these groups of patients.

SDB implies testing during sleep, including laboratory polysomnography (PSG) or home sleep apnea testing (5). The association between HF, sleep apnea, and rhythm disturbances has been analyzed, revealing the coexistence of these pathologies in $>50 \%$ of affected individuals $(6,7)$.

Still, the complex interrelation between HF, SDB, and cardiac rhythm disturbances are poorly studied, especially when considering the individual prevalence and mechanism of different types of sleep apnea. It is interesting that OSA has a rather low incidence and prevalence in $\mathrm{HF}$, variables that are merely comparable with the ones in the general population, ranging from $8 \%$ in the study by Grimm et al. (7) to $38 \%$ in the study by Sin et al. (8).

In the same contex, Sin et al. (8) included 450 patients with $\mathrm{HF}$ in the study, all of whom were referred to the sleep laboratory because of suspected SDB. This distinct randomization explains the high variability of the obtained data. Oldenburg et al. (9) confirmed the high prevalence of SDB, particularly CSA in CHF patients, CSA resembled to be a marker of heart failure severity. This important difference between the data may be explained by the different criteria of inclusion. Grimm et al. (10) selected patients with HF after patients with a history of SDB and with symptoms suggestive of SDB had been excluded.

At the same time, CSA has a notably higher frequency of about $50 \%$ in patients with $\mathrm{HF}$, ranging from $21 \%$ to $82 \%$ according to different data sources (3).

Despite these data, it is yet unclear whether SDB is an independent marker of an eventual poor outcome or an independent prognostic factor in HF. There is a high certainty that SDB involves a high risk for supraventricular and ventricular arrhythmias (10).

Otherwise, concerning the type of $\mathrm{HF}$, there is a large amount of data referring to the association of SDB with arrhythmias and $\mathrm{HF}$ with reduced ejection fraction (HF-rEF), whereas the association between SDB, arrhythmias, and $\mathrm{HF}$ with preserved ejection
(HF-pEF) is poorly reported, with the prevalence of CSA reported as approximately $30 \%$ in this group, whereas the prevalence of SDB in HF with mid-range ejection fraction (HF-mrEF) is lacking consistent data.

Anyway, although there are many subjects with an inconsistency of data and evidence, there is one certain thing: regardless of the nature of SDB (whether OSA or CSA), suspicion for sleep apnea should be high in patients with $\mathrm{HF}$ because approximately two thirds of these have sleep apnea.

\section{CSA and HF}

$\mathrm{HF}$ is a major health issue with an increasing incidence, a high rate of hospitalizations, and high mortality in developing countries. Focusing on early recognition and management of $\mathrm{HF}$ comorbidities may have an important role in reducing the economic burden and public health impact of HF (5).

CSA is associated with a significant risk of increased morbidity and mortality in patients with $\mathrm{HF}$, resulting in intermittent hypoxemia, excessive activity of the renin-angiotensin-aldosterone system, and worsening of HF signs and symptoms (11).

The characteristics of patients with CSA are different when compared with the characteristics of those presenting with OSA. The majority of patients with CSA and HF are middle-aged and older males with a normal or nearly normal body mass index (BMI), presenting with arrhythmia (atrial and ventricular), fatigue, nycturia, and daytime hypocapnia [partial pressure of carbon dioxide $\left(\mathrm{CO}_{2}\right)<38 \mathrm{~mm} \mathrm{Hg}$ ] and are known to have reduced ejection fraction and recurrent HF hospitalizations. Snoring is not very usual as it is in patients with OSA. It would be obvious to think that these patients should have daytime symptoms and be aware of the sleep disturbance they have, especially those presenting with severe forms of CSA, but surprisingly, a great number of them do not experience daytime fatigue, sleepiness, or disordered sleep. There are several hypotheses explaining this fact. One of these hypotheses suggests the poor QoL of this category of patients with $\mathrm{HF}$ and the fatigue characterizing the underlying disease. Another hypothesis reports the potential increase in sympathetic activity, which effaces the typical sleepiness symptoms.

Usually, CSA occurs in one of the two classical forms: Cheyne-Stokes respiration or non-Cheyne-Stokes respiration, with a higher incidence of the Cheyne-Stokes pattern in patients with $\mathrm{HF}$, resulting from a delay in the respiratory control center in detecting and responding to changes in $\mathrm{CO}_{2}$ above and below the apneic threshold (12).

CSA may be considered a marker of advanced HF, often being associated with elevated pulmonary capillary wedge pressure, BNP and noradrenaline levels and low left ventricular ejection fraction (LVEF). In the same way, there is an important correlation between CSA and the different types of arrhythmias, which require rhythm correction, pacemakers (atrial overdrive or biventricular) (11-13), or other types of interventions with a life-saving impact and, most importantly, an influence on 0oL. 
Here comes the essential importance of the early detection and correction of rhythm disturbances in patients with HF and CSA (14).

\section{CSA, HF, and cardiac arrhythmias}

The correlation between SDB, HF, and cardiac arrhythmias has been analyzed in several studies. One of those is the MrOS Sleep Study that studied the association between SDB with nocturnal atrial fibrillation (AF) or flutter and complex ventricular ectopy in older men. An extensive analysis in this study showed a progressively increased frequency of $\mathrm{AF}$ and complex ventricular events along with increasing severity of SDB.

In addition, a detailed series of analyses investigating the relative contribution of OSA and CSA to these associations indicated that the relationship between SDB and nocturnal AF was largely associated with CSA but not purely with OSA. On the other hand, ventricular arrhythmias were associated with OSA but not with CSA and were also associated with overnight hypoxia. HF is a factor that may precipitate CSA by mechanisms of increased respiratory chemoreceptor drive, pulmonary congestion and/or hyperventilation, and prolonged circulation time; therefore, the analyses adjusted for self-reported HF showed an approximately three-fold increased odds of AF in association with CSA and fivefold increased odds of AF with the Cheyne-Stokes form of CSA. It is possible that these relationships could be the result of unrecognized HF or be related to underlying structural heart disease not measured in this study, such as ventricular hypertrophy and atrial enlargement. When SDB was characterized according to central or obstructive subtypes, complex ventricular ectopy was associated most strongly with OSA and hypoxia, whereas AF was most strongly associated with CSA, suggesting that different sleep-related stresses may contribute to atrial and ventricular arrhythmogenesis in older men (15). Meanwhile, the results of the Dry Eye Assessment and Management study showed that obstructive respiratory events were stronger predictors of nocturnal cardiac arrhythmias than central respiratory events.

Various measures of hypoxemia were significantly associated with nocturnal cardiac arrhythmia, whereas the arousal index-a measure of arousal from sleep (and presumably sympathetic activity) - was not, associations found in a sleep clinicbased population of U.S. veterans at risk of developing both SDB and cardiac arrhythmias. Regarding conduction delay arrhythmias, the prevalence of sinus pauses, atrioventricular block, and asystole has been reported in up to $18 \%$ of patients with SDB, in comparison with just $3 \%$ in a healthy population. In patients with sleep apnea, the occurrence of conduction delay arrhythmias has been associated with SDB severity, the extent of hypoxemia, and REM sleep. The study found a significant relationship of hypoxemia with the frequency of intraventricular conduction delay. In contrast to these findings, Mehra et al. (16) reported no significant difference in conduction delay arrhythmia between individuals with SDB and those without. This difference in observational data may be explained by differences in study design and study population as well as in the degree of oxygen desaturation. The combination of an apneic event and hypoxemia is necessary to trigger a vagally mediated apneic arrhythmia (17).

There is emerging evidence linking CSA and AF, and CSA may be a marker of abnormal autonomic function, respiratory chemoreflex sensitivity, and cardiac function. Although studies performed to date have provided some insight into the mechanism underlying these conditions, more investigation is needed to understand the clinical overlap between these common clinical entities. Considering that nearly one quarter of those with CSA and $30 \%$ of those with Cheyne-Stokes form of CSA developed $A F$, the presence of CSA or Cheyne-Stokes respiration may be used to target individual patients for $\mathrm{AF}$ risk reduction. Sleep Heart Health Study (SHHS) assessed the association of OSA and CSA with incident $A F$ among 2,912 individuals without a history of $A F$, concluding that CSA has been associated with AF in patients with HF (18).

\section{Extended cardiac monitoring in CSA}

The large intraindividual rhythm variability was reported by Simantirakis et al. (18) in a cohort of patients with moderate to severe OSA who underwent continuous monitoring by implantable loop recorder (ILR), showing the incapability of 24-hour and 48-hour Holter monitoring to accurately determine the incidence of atrial arrhythmias. Therefore, assuming that continuous electrocardiographic (ECG) monitoring is the most reliable tool to determine the incidence of cardiac arrhythmias, approximately half of patients with OSA present with severe cardiac rhythm disturbances. Moreover, the frequency and severity of apnearelated nocturnal bradyarrhythmias correlate with BMI, apneahypopnea index (AHI), and desaturation level during the sleep study (19-21). On the other hand, in a smaller prospective study, which analyzed the AF recurrence profile after the first clinical episode and investigated the clinical parameters associated with the course of arrhythmia using an ILR, there was no significant percentage of patients with AF recurrence after their first symptomatic episode or after they showed a low recurrence rate. Most patients presented with episodes of short duration, but these results must be confirmed by larger studies (22).

More accurate follow-up using ILRs shows that the initiation of continuous positive airway pressure (CPAP) therapy tends to reduce the total number of recorded episodes in the short term, whereas in the long-term, bradycardia episodes are completely abolished. CPAP therapy also has the capability to decrease the OT-corrected interval dispersion present at baseline in patients with moderate to severe OSA (23). The latter might have implications in preventing the related bradycardia early after depolarizations and the ventricular arrhythmias leading to nocturnal sudden death (24).

In elderly patients, only CSA but not OSA was associated with the presence of $A F$; moreover, prolonged hypoxia during sleep and the overall severity of SDB are associated with AF in $\operatorname{SDB}(25,26)$. 
The presence of CSA is associated with $\mathrm{HF}$ in the elderly, which is a well-known risk factor for AF (27). In addition, several well-recognized risk factors for the burden of $A F$, such as atrial enlargement, valvular diseases, and left ventricular function, have generally not been considered in large cohort studies, which may have biased their conclusions. In another trial, the severity of CSA and increased levels of C-reactive protein are associated with the presence of $A F$ in patients with $\mathrm{HF}(28)$.

Bitter et al. (29) studied the association of CSA with an increased risk of malignant cardiac arrhythmias in patients with congestive HF, showing an independent correlation of CSA with a shorter event-free survival period, awaiting first monitored ventricular arrhythmia, and appropriate cardioverter-defibrillator therapy in patients with HF.

Although the correlation between CSA and AF has been repeatedly observed, the optimal treatment of $A F$ in patients with CSA has not been extensively investigated, which is most likely due to the common coexistence of CSA and HF. The presence of $\mathrm{HF}$ per se reduces the success of external cardioversion and ablation, which may be an important confounder in a study design (30).

Despite the strong evidence that CSA is an important treatable risk factor for AF, stroke, and death, CSA is not included in any conventional multiparametric risk scores. Patients with AF with coexistent SDB, especially those with a moderate to severe $\mathrm{AHI}$, should be considered at high risk of ischemic events. This is why it is very important to identify the different types of cardiac arrhythmia in patients with SDB, especially in those with CSA, which is highly associated with $\mathrm{HF}$ and worsens the prognosis of these patients with an important increase in mortality and morbidity (31).

Apnea-associated arrhythmia may contribute to sudden cardiac death and premature mortality in HF. CSA exerts strong modulatory effects on the autonomic system with a special autonomic profile. Profound vagal activity leads to bradyarrhythmias, and sympathetic excitation leads to tachyarrhythmias. AF and ventricular arrhythmias in patients with CSA are mainly found in combination with cardiovascular comorbidity (32).

The mechanisms and pathophysiology of the connection between CSA and supraventricular and ventricular arrhythmias are still under-recognized, although some of them have already been studied, explained, and described by researchers, but in any case, the necessity of a deep, multidisciplinary approach for the identification of all the miscellaneous masks of this tricky pathology is obvious.

The Extended Cardiac Monitoring in Patients with Severe Sleep Apnea and No History of Atrial Fibrillation Study had the aim to determine the incidence of new-onset $A F$ in patients with severe OSA syndrome by the use of an ILR, emphasizing the increasing role of the extended ECG monitoring in the diagnostic work-up and management of patients with SDB. The results of the study showed that $20 \%$ of patients with severe OSA had newonset $A F$, emphasizing the fact that extended cardiac monitoring of patients with severe OSA may facilitate the identification of new-onset arrhythmias (32). Similar results were described by the REVEAL AF Study, which enrolled 446 patients at high risk for but without previously known AF using an insertable cardiac monitor, including 104 patients $(26.4 \%)$ with sleep apnea and 81 patients $(20.6 \%)$ with $\mathrm{HF}$, with an important overlap between these two diseases in the study population and a maximal follow-up of 30 months. The AF detection rate was $6.2 \%$ at 30 days, $20.4 \%$ at 6 months, $27.1 \%$ at 12 months, $33.6 \%$ at 24 months, and $40.0 \%$ at 30 months; most patients would not have been identified with shorter duration monitoring typical of external devices.

Two smaller studies-Prevalence of Sub-Clinical Atrial Fibrillation Using an Implantable Cardiac Monitor (ASSERT-II) study and The Predicting Atrial Fibrillation or Flutter (PREDATEAF) study - have also reported on the incidence of AF in highrisk populations using implantable recorders. The rate of detection of AF lasting $\geq 5$ minutes was $34.4 \%$ per person-year in the ASSERT-II study, and the incidence of AF or atrial flutter was $22 \%$ during an average follow-up of approximately 15 months in the PREDATE-AF study $(33,34)$.

The LOOP study is an ongoing randomized controlled trial with 6,000 participants to receive an ILR with continuous ECG monitoring. Participants included are aged $\geq 70$ years and were previously diagnosed as having at least one of the following conditions: hypertension, diabetes mellitus, $\mathrm{HF}$, or previous stroke. The primary endpoint is time to stroke or systemic embolism, whereas secondary endpoints include time to AF diagnosis and death. The study is expected to evaluate the health benefits and cost effectiveness of ILR as a screening tool for AF to prevent stroke in patients at risk as well as identify the risk factors for the development of AF and characterization of arrhythmias in the high-risk population $(35,36)$.

These results emphasize the impact of extended cardiac monitoring using ILR in the diagnosis of $A F$ in high-risk patients, including patients with HF and SDB with or without symptoms, with important implications in screening and stroke prevention in this population (37).

Most patients with HF have SDB, and with CSA rather than OSA becoming the predominant form in patients with more severe disease, these patients have a worse prognosis than those without SDB. Mask-based therapies targeted for SDB can improve sleep quality and can partially normalize sleep and respiratory physiology. However, recent randomized trials of cardiovascular outcomes in CSA in chronic HF-rEF have had neutral findings or suggested the possibility of harm, likely from an increased rate of sudden death, meaning that surrogate endpoints of improvement in respiratory and sleep metrics can no longer be taken as adequate therapeutic outcome measures in patients with HF and sleep apnea (38).

The impact of implantable devices in patients with sleep apnea was also revealed and underlined in a study that included 160 patients with a dual-chamber pacemaker. The results of the study showed an independent association of severe forms of sleep apnea with a higher risk of AF and new-onset AF (39). 
Different types of arrhythmias were noted to be associated with SDB and HF. Bradycardia and atrioventricular block are mediated by vagal stimulation accompanying apneas and hypopneas, the latter tending to occur during REM sleep and improve with CPAP treatment.

The majority of patients with persistent AF have been shown to have SDB. Among the participants in the SHHS, moderate to severe SDB was associated with a four-fold increase in the odds of AF. Data from a meta-analysis reported that the risk ratio for recurrent $A F$ after pulmonary vein isolation was 1.25 in patients with OSA but not CSA compared with the ratio in those without OSA. In AF management guidelines, OSA is mentioned as being associated with $\mathrm{AF}$ and as a factor contributing to a reduction in the success of ablation procedures (40). Consideration of screening for sleep apnea in such patients is reasonable, and if OSA is diagnosed, treatment with CPAP may maximize the effectiveness of other rhythm-control strategies; these data are inconclusive for CSA. A high frequency of AF after coronary artery bypass graft surgery also has been reported in patients with SDB compared with the frequency in patients without $S D B$, suggesting the importance of untreated SDB in modifying postcardiac surgery cardiovascular outcomes. Concerning ventricular arrhythmias, patients with HF and SDB had a three-fold increased odds of nonsustained ventricular tachycardia and almost twice the risk of complex ventricular ectopy on PSG in the SHHS. Improvement in ventricular ectopy with CPAP treatment has been shown in patients with SDB and HF. An AHI >20 per hour was a significant and independent risk factor for incident sudden cardiac death in a study of $>10,000$ patients with HF referred for PSG (41). Coexisting HF and SDB (both OSA and CSA) increase the risk of developing malignant ventricular arrhythmia in patients with implanted cardioverter defibrillators (42).

Registry data show that treatment of SDB with Adaptive Servo-Ventilation (ASV) in patients with HF who have implantable cardioverter-defibrillator devices (ICDs) is associated with lesser use of defibrillatory therapies and improvements in cardiac function and respiratory stability. However, in the light of the increased mortality in a large randomized trial of ASV in patients with HF-rEF and predominantly CSA (The Treatment of Predominant Central Sleep Apnoea by Adaptive Servo Ventilation in Patients With Heart Failure study) (43), mask-based therapy for CSA should be avoided at the present time.

A meta-analysis of nine prospective cohort studies published between 1999 and 2013 with a total of 1,274 patients assessed the clinical impact of SDB on the incidence of appropriate ICD therapy, a surrogate for malignant ventricular arrhythmia. The results of the study showed that in patients with HF-rEF, the risk of appropriate ICD therapy is higher among patients with SDB than among those without. CSA is a well-established risk factor for high mortality in HF, and the finding of increased risk of ICD therapy in patients with CSA suggests that malignant arrhythmia may be one of the mechanisms of mortality in patients with HF who have CSA. At the same time, patients with SDB and underly- ing HF may also be subject to serious bradyarrhythmias in sleep. Nocturnal bradyarrhythmias, including prolonged sinus pauses occurring during apneic episodes, are much more prevalent in patients with CSA than in those without, and although these arrhythmias have been generally considered benign, the prevalence and its potentially deleterious impact on the outcome of patients with HF in association with SDB may need deeper research. Excessive parasympathetic response to apneic respiratory events is thought to be an underlying mechanism for bradyarrhythmias; this response is exaggerated by impaired baroreflexes, which is frequently encountered in HF.

Therefore, using ICD and ILR would enlighten the grey zones of this subject, confirming the high risk of life-threatening arrhythmias in patients with HF in association with CSA (44).

\section{The role of ILR in detecting ventricular arrhythmia in patients with HF}

Although it is increasingly recognized that noncardiovascular death is more common in patients with HF-mrEF and HF-pEF than in patients with HF with reduced LVEF, sudden death is still one of the most common causes of death in this population. It is unclear how many of these patients die from an arrhythmia and, if so, whether these are tachyarrhythmias or bradyarrhythmias. The Ventricular Tachyarrhythmia Detection by Implantable Loop Recording in Patients with Heart Failure and Preserved Ejection Fraction study (VIP-HF study) is a prospective study in patients with HF-mrEF/HF-pEF attempting to determine the incidence of ventricular tachyarrhythmias and rhythm directly before sudden death. In the VIP-HF study, sustained ventricular tachyarrhythmias and sudden death were uncommon. The prediction of increased risk of sudden death in patients with HF-mrEF/HF-pEF has been shown to be difficult if not impossible. Attempts to create risk prediction models in patients with LVEF $>40 \%$ found age, male sex, history of myocardial infarction and diabetes, presence of left bundle branch block, and NT-proBNP as risk indicators of sudden death (45). In addition, earlier studies suggest that the mortality risk may be greater in ischaemic causes of HF than HF due to nonischaemic causes. The majority of patients in the VIP-HF study did not have a previous myocardial infarction, which may have led to a lower sudden death rate. In a recent paper focusing on renal patients, it was shown that in contrast to traditional thinking, sudden death is often not caused by ventricular tachyarrhythmias but rather by bradyarrhythmias followed by asystole. Interestingly, in the VIP-HF study, we also observed a higher incidence of bradyarrhythmias than of sustained ventricular tachyarrhythmias. One study examined the value of ILR in patients with reduced LVEF and found clinically significant bradyarrhythmias in a substantial proportion of patients (46).

To our knowledge, no data on the incidence of bradyarrhythmias in HF-pEF are currently available. The use of an ILR led to an increased number of patients in whom nonsustained ventricular tachyarrhythmias were detected (from $18 \%$ on baseline 24 -hour Holter monitoring to $27 \%$ ). No association between 
nonsustained ventricular tachyarrhythmias on Holter monitoring and HF hospitalization or mortality was observed. In a recent retrospective study of 85 patients with HF-pEF who have a pacemaker, $45 \%$ of patients had episodes of nonsustained ventricular tachyarrhythmias during 4 years of follow-up (47).

Also in this study, no association was found between nonsustained ventricular tachyarrhythmias and mortality. Patients with $\mathrm{HF}$ and a pacemaker are not comparable with those without a pacemaker because their prognosis is more impaired. Establishing the cause of death with an ILR may therefore be difficult because the recording of the causative episode may be replaced after death with newer recordings of asystole or noise. The absence of intracardiac leads, making a distinction between low-amplitude ventricular fibrillation and asystole difficult, also increases the risk for noise capture.

\section{Conclusions and further directions}

Taking into consideration the inconsistency of data and lack of good quality evidence concerning the management of CSA in patients with HF and the high rate of arrhythmic morbidity progressively rising along with the $\mathrm{HF}$ and CSA severity increase as well as the high mortality by arrhythmic sudden cardiac death, it becomes imperative for early detection of cardiac arrhythmias in these groups of patients.

HF can worsen progressively over days or weeks, but current telemedicine systems might not be sufficient to detect acute exacerbations in $\mathrm{HF}$ or to prevent rehospitalization (48). Conversely, arrhythmias can often occur suddenly or intermittently and might require immediate intervention $(49,50)$. Advances in cardiovascular monitoring technologies, such as the use of ubiquitous mobile devices and the development of novel portable sensors with seamless wireless connectivity and machine learning algorithms that can provide a specialist-level diagnosis in near real time, have the potential for a more personalized care. Devices have been developed to assess hemodynamics, which can detect potential signs of worsening HF (49). Furthermore, continuous ECG recordings have been used to redefine phenotypes for $A F$ and ventricular arrhythmias (50) and can predict the success of antiarrhythmic therapy (51). Wearable activity trackers and smartwatches can measure physiological indices, such as heart rate, breathing patterns, and cardiometabolic activity (52), and can even detect AF. Furthermore, smartphone applications have been successful in shortening the time to first response for sudden cardiac arrest. This confluence of novel technologies has also attracted much public interest and the promise to expand applications for cardiovascular monitoring. The advantages of ILRs include the capacity for long-term monitoring and consistent ECG wave morphologies owing to a fixed spatial orientation. Paradoxically, such devices are suboptimal for the diagnosis of arrhythmias of short durations (tens of seconds to minutes) and for classifying the type of atrial arrhythmia (53). These limitations might be overcome with improvements in signal processing algorithms (54). Finally, numerous wearable ECG devices are available to the public. The Apple Watch (Apple, USA) and KardiaMobile (Alivecor, USA) are approved by the Food and Drug Administration for rhythm monitoring and have clinical-level accuracy for the detection of arrhythmias, such as AF (55). None of these devices provides continuous monitoring, although daily and nightly use for months might ultimately provide near-continuous recordings. However, at present, these devices require activation by the patient to record the ECG, and smartwatch pulse checks (through photoplethysmography) occur only intermittently. Therefore, these monitors can miss paroxysmal arrhythmic events that are too short in duration or too catastrophic in nature to be captured by the patient and cannot measure arrhythmia burden. As wearable devices become increasingly flexible, stretchable, and weightless, they can be comfortably worn continuously to provide uninterrupted ECG data.

The major limitation of external loop recorders (ELR) for the diagnosis of unpredictable and infrequent symptoms, such as syncope, is that the patients must wear continuously external electrodes in order to activate loop memory. The ELR appears to have its greatest role in motivated patients with frequent (pre) syncopes where spontaneous symptoms are likely to recur within 4-6 weeks. This time frame is usually the maximum that a patient can comply with; shorter durations yield lower diagnostic yield. In randomized comparison studies, ELRs proved to yield a higher diagnostic value than conventional evaluation or Holter monitoring but a lower value than ILRs (56).

ILRs have the best yield (up to a $75 \%$ detection rate for the cause of syncope at 3 years). They do not need to be removed during certain activities, such as showering or swimming, and may identify significant cardiac rhythm abnormalities when the patient is sleeping. Patients can freely continue with their normal activities of everyday living and exercise. An identification card is usually given to the patient with the make and model of their ILR device. This information is valuable should the patient with recurrent symptoms present to the emergency department and need to have their device checked. The main purpose of the device is to record electrical activity and is therefore safe to travel with, and it is not affected by other electrical devices. Event recorders are the next most productive, and Holter monitors are the least effective unless events occur daily, which is not the case for most patients (57).

After the monitoring period, there are three possible combinations of patient symptoms and ECG findings. The first possibility is when the patient has symptoms with corresponding ECG abnormalities, in which case appropriate therapy should be initiated. Possible recommendations include implantation of a permanent pacemaker, implantation of an implantable cardiac defibrillator, or a diagnostic electric study of the heart that may or may not be followed by a therapeutic procedure. If an arrhythmia is detected, such as AF, the patient may need to start a bloodthinning medication to decrease the risk of a cerebral vascular accident. The second possibility is when the patient has symptoms without corresponding ECG abnormalities, in which case 
the diagnosis is unlikely cardiac in nature, and other etiologies for the patient's symptoms should be investigated. The patient can be referred to other more appropriate specialists if necessary. The third possibility is when the patient has no symptoms and no ECG abnormalities during the monitoring period, and a cardiac etiology is unlikely, and no further ECG monitoring is required. If a diagnosis is made or no abnormalities were discovered during the monitoring period, the patient can have the device removed in a similar fashion that it was implanted (56).

Although the relationship between CSA, cardiac arrhythmias, and conduction disturbances has been analyzed in several studies, the arrhythmic implication of the CSA in adults with HF remains controversial. Taking into consideration the high rate of asymptomatic arrhythmias but with high mortality risk, especially concerning ventricular arrhythmias, bradyarrhythmias, and different types of heart blocks in patients with HF and CSA, the extended cardiac monitoring by ILR becomes compulsory in these groups of patients because in-time interventions could be life saving for them, but with the absolute lack of solid evidence in this field, there is an acute need of extensive randomized trials to further highlight the potential beneficial effect of ILR monitoring in patients with CSA and HF.

\section{Conflict of interest: None declared.}

Peer-review: Externally peer-reviewed.

Author contributions: Concept - I.C.P., V.R.; Design - I.C.P.; Supervision - V.R.; Fundings - None; Materials - I.C.P.; Data collection \&/or processing - I.C.P.; Analysis \&/or interpretation - I.C.P.; Literature search - I.C.P.; Writing - I.C.P.; Critical review - V.R.

\section{References}

1. Bekfani T, Abraham WT. Current and future developments in the field of central sleep apnoea. Europace 2016; 18: 1123-34. [Crossref]

2. Naughton MT. Respiratory sleep disorders in patients with congestive heart failure. J Thorac Dis 2015; 7: 1298-310.

3. Kapur VK, Auckley DH, Chowdhuri S, Kuhlmann DC, Mehra R, Ramar K, et al. Clinical Practice Guideline for Diagnostic Testing for Adult Obstructive Sleep Apnea: An American Academy of Sleep Medicine Clinical Practice Guideline. J Clin Sleep Med 2017; 13: 479-504. [Crossref]

4. Korostovtseva LS, Zvartau NE, Rotar OP, Sviryaev YV, Konradi AO. Predictors of heart rhythm disturbances in hypertensive obese patients with obstructive sleep apnea. J Geriatr Cardiol 2017; 14: 553-62.

5. Resano-Barrio MP, Arroyo-Espliguero R, Viana-Llamas MC, Mediano 0. Obstructive Sleep Apnoea Syndrome: Continuous Positive Airway Pressure Therapy for Prevention of Cardiovascular Risk. Eur Cardiol 2020; 15: e65. [Crossref]

6. Szymański FM, Płatek AE, Karpiński G, Koźluk E, Puchalski B, Filipiak KJ. Obstructive sleep apnoea in patients with atrial fibrillation: prevalence, determinants and clinical characteristics of patients in Polish population. Kardiol Pol 2014; 72: 716-24. [Crossref]
7. Grimm W, Sass J, Sibai E, Cassel W, Hildebrandt 0, Apelt S, et al. Severe central sleep apnea is associated with atrial fibrillation in patients with left ventricular systolic dysfunction. Pacing Clin Electrophysiol 2015; 38: 706-12. [Crossref]

8. Sin DD, Fitzgerald F, Parker JD, Newton G, Floras JS, Bradley TD. Risk factors for central and obstructive sleep apnea in 450 men and women with congestive heart failure. Am J Respir Crit Care Med 1999; 160: 1101-6. [Crossref]

9. Oldenburg 0 , Lamp B, Faber L, Teschler H, Horstkotte D, Töpfer V. Sleep-disordered breathing in patients with symptomatic heart failure: a contemporary study of prevalence in and characteristics of 700 patients. Eur J Heart Fail 2007; 9: 251-7. [Crossref]

10. Grimm W, Koehler U. Cardiac arrhythmias and sleep-disordered breathing in patients with heart failure. Int $\mathrm{J}$ Mol Sci 2014; 15: 18693-705. [Crossref]

11. Naughton MT, Benard DC, Liu PP, Rutherford R, Rankin F, Bradley TD. Effects of nasal CPAP on sympathetic activity in patients with heart failure and central sleep apnea. Am J Respir Crit Care Med 1995; 152: 473-9. [Crossref]

12. Solin P, Kaye DM, Little PJ, Bergin P, Richardson M, Naughton MT. Impact of sleep apnea on sympathetic nervous system activity in heart failure. Chest 2003; 123: 1119-26. [Crossref]

13. Harun NS, Leet A, Naughton MT. Improvement in sleep-disordered breathing after insertion of left ventricular assist device. Ann Am Thorac Soc 2013; 10: 272-3. [Crossref]

14. Aurora RN, Chowdhuri S, Ramar K, Bista SR, Casey KR, Lamm Cl, et al. The treatment of central sleep apnea syndromes in adults: practice parameters with an evidence-based literature review and meta-analyses. Sleep 2012; 35: 17-40. [Crossref]

15. Mehra R, Stone KL, Varosy PD, Hoffman AR, Marcus GM, Blackwell $T$, et al. Nocturnal Arrhythmias across a spectrum of obstructive and central sleep-disordered breathing in older men: outcomes of sleep disorders in older men (MrOS sleep) study. Arch Intern Med 2009; 169: 1147-55. [Crossref]

16. Mehra R, Benjamin EJ, Shahar E, Gottlieb DJ, Nawabit R, Kirchner $\mathrm{HL}$, et al.; Sleep Heart Health Study. Association of nocturnal arrhythmias with sleep-disordered breathing: The Sleep Heart Health Study. Am J Respir Crit Care Med 2006; 173: 910-6. [Crossref]

17. Selim BJ, Koo BB, Oin L, Jeon S, Won C, Redeker NS, et al. The Association between Nocturnal Cardiac Arrhythmias and SleepDisordered Breathing: The DREAM Study. J Clin Sleep Med 2016; 12: 829-37. [Crossref]

18. Simantirakis EN, Schiza SI, Marketou ME, Chrysostomakis SI, Chlouverakis GI, Klapsinos NC, et al. Severe bradyarrhythmias in patients with sleep apnoea: the effect of continuous positive airway pressure treatment: a long-term evaluation using an insertable loop recorder. Eur Heart J 2004; 25: 1070-6. [Crossref]

19. Becker HF, Koehler U, Stammnitz A, Peter JH. Heart block in patients with sleep apnoea. Thorax 1998; 53 (Suppl 3): S29-32. [Crossref]

20. Hoffstein V, Mateika S. Cardiac arrhythmias, snoring, and sleep apnea. Chest 1994; 106: 466-71. [Crossref]

21. Simantirakis EN, Papakonstantinou PE, Kanoupakis E, Chlouverakis GI, Tzeis S, Vardas PE. Recurrence rate of atrial fibrillation after the first clinical episode: A prospective evaluation using continuous cardiac rhythm monitoring. Clin Cardiol 2018; 41: 594600. [Crossref]

22. Dursunoglu D, Dursunoglu N. Effect of CPAP on QT interval dispersion in obstructive sleep apnea patients without hypertension. Sleep Med 2007; 8: 478-83. [Crossref] 
23. Patel D, Mohanty P, Di Biase L, Shaheen M, Lewis WR, Quan K, et al. Safety and efficacy of pulmonary vein antral isolation in patients with obstructive sleep apnea: the impact of continuous positive airway pressure. Circ Arrhythm Electrophysiol 2010; 3: 445-51. [Crossref]

24. Urbanik D, Martynowicz H, Mazur G, Poręba R, Gać P. Environmental Factors as Modulators of the Relationship between Obstructive Sleep Apnea and Lesions in the Circulatory System. J Clin Med 2020; 9: 836. [Crossref]

25. Arzt M, Floras JS, Logan AG, Kimoff RJ, Series F, Morrison D, et al.; CANPAP Investigators. Suppression of central sleep apnea by continuous positive airway pressure and transplant-free survival in heart failure: a post hoc analysis of the Canadian Continuous Positive Airway Pressure for Patients with Central Sleep Apnea and Heart Failure Trial (CANPAP). Circulation 2007; 115: 3173-80. [Crossref]

26. Gami AS, Hodge D0, Herges RM, Olson EJ, Nykodym J, Kara T, et al. Obstructive sleep apnea, obesity, and the risk of incident atrial fibrillation. J Am Coll Cardiol 2007; 49: 565-71. [Crossref]

27. Sano K, Watanabe E, Hayano J, Mieno Y, Sobue $Y$, Yamamoto M, et al. Central sleep apnoea and inflammation are independently associated with arrhythmia in patients with heart failure. Eur $\mathrm{J}$ Heart Fail 2013; 15: 1003-10. [Crossref]

28. Kendzerska T, Mollayeva T, Gershon AS, Leung RS, Hawker G, Tomlinson $G$. Untreated obstructive sleep apnea and the risk for serious long-term adverse outcomes: a systematic review. Sleep Med Rev 2014; 18: 49-59. [Crossref]

29. Bitter T, Westerheide N, Prinz C, Hossain MS, Vogt J, Langer C, et al. Cheyne-Stokes respiration and obstructive sleep apnoea are independent risk factors for malignant ventricular arrhythmias requiring appropriate cardioverter-defibrillator therapies in patients with congestive heart failure. Eur Heart J 2011; 32: 61-74. [Crossref]

30. Padeletti M, Zacà V, Mondillo $S$, Jelic S. Sleep-disordered breathing increases the risk of arrhythmias. J Cardiovasc Med (Hagerstown) 2014; 15: 411-6. [Crossref]

31. Koehler U, Reinke C, Sibai E, Hildebrandt O, Sohrabi K, Dette F, et al. Autonomic dysfunction and cardiac arrhythmia in patients with obstructive and central sleep apnea. Dtsch Med Wochenschr 2011; 136: 2622-8. [Crossref]

32. Yeung C, Drew D, Hammond S, Hopman WM, Redfearn D, Simpson C, et al. Extended Cardiac Monitoring in Patients With Severe Sleep Apnea and No History of Atrial Fibrillation (The Reveal XT-SA Study). Am J Cardiol 2018; 122: 1885-9. [Crossref]

33. Van Gelder IC, Healey JS, Crijns HJGM, Wang J, Hohnloser SH, Gold MR, et al. Duration of device-detected subclinical atrial fibrillation and occurrence of stroke in ASSERT. Eur Heart J 2017; 38: 1339-44. [Crossref]

34. Nasir JM, Pomeroy W, Marler A, Hann M, Baykaner T, Jones R, et al. Predicting Determinants of Atrial Fibrillation or Flutter for Therapy Elucidation in Patients at Risk for Thromboembolic Events (PREDATE AF) Study. Heart Rhythm 2017; 14: 955-61. [Crossref]

35. Diederichsen SZ, Haugan KJ, Køber L, Højberg S, Brandes A, Kronborg $C$, et al. Atrial fibrillation detected by continuous electrocardiographic monitoring using implantable loop recorder to prevent stroke in individuals at risk (the LOOP study): Rationale and design of a large randomized controlled trial. Am Heart J 2017; 187: 122-32. [Crossref]

36. Leggio M, Lombardi M, Caldarone E, D’Emidio S, Severi P, Armeni $M$, et al. Pacemaker-detected severe sleep apnoea predicts newonset atrial fibrillation. Europace 2018; 20: 2046-7. [Crossref]
37. Cowie MR, Gallagher AM. Sleep Disordered Breathing and Heart Failure: What Does the Future Hold? JACC Heart Fail 2017; 5: 71523. [Crossref]

38. Calkins H, Kuck KH, Cappato R, Brugada J, Camm AJ, Chen SA, et al.; Heart Rhythm Society Task Force on Catheter and Surgical Ablation of Atrial Fibrillation. 2012 HRS/EHRA/ECAS expert consensus statement on catheter and surgical ablation of atrial fibrillation: recommendations for patient selection, procedural techniques, patient management and follow-up, definitions, endpoints, and research trial design: a report of the Heart Rhythm Society (HRS) Task Force on Catheter and Surgical Ablation of Atrial Fibrillation. Developed in partnership with the European Heart Rhythm Association (EHRA), a registered branch of the European Society of Cardiology (ESC) and the European Cardiac Arrhythmia Society (ECAS); and in collaboration with the American College of Cardiology (ACC), American Heart Association (AHA), the Asia Pacific Heart Rhythm Society (APHRS), and the Society of Thoracic Surgeons (STS). Endorsed by the governing bodies of the American College of Cardiology Foundation, the American Heart Association, the European Cardiac Arrhythmia Society, the European Heart Rhythm Association, the Society of Thoracic Surgeons, the Asia Pacific Heart Rhythm Society, and the Heart Rhythm Society. Heart Rhythm 2012; 9: 632-96. [Crossref]

39. Gami AS, Olson EJ, Shen WK, Wright RS, Ballman KV, Hodge DO, et al. Obstructive sleep apnea and the risk of sudden cardiac death: a longitudinal study of 10,701 adults. J Am Coll Cardiol 2013; 62: 610-6. [Crossref]

40. Bitter T, Westerheide N, Hossain SM, Prinz C, Horstkotte D, Oldenburg 0 . Symptoms of sleep apnoea in chronic heart failure--results from a prospective cohort study in 1,500 patients. Sleep Breath 2012; 16: 781-91. [Crossref]

41. Cowie MR, Woehrle H, Wegscheider K, Angermann C, d'Ortho MP, Erdmann E, et al. Adaptive Servo-Ventilation for Central Sleep Apnea in Systolic Heart Failure. N Engl J Med 2015; 373: 1095-105. [Crossref]

42. Cowie MR. Sleep apnea: State of the art. Trends Cardiovasc Med 2017; 27: 280-9. [Crossref]

43. Kwon Y, Koene RJ, Kwon O, Kealhofer JV, Adabag S, Duval S. Effect of Sleep-Disordered Breathing on Appropriate Implantable Cardioverter-Defibrillator Therapy in Patients With Heart Failure: A Systematic Review and Meta-Analysis. Circ Arrhythm Electrophysiol 2017; 10: e004609. [Crossref]

44. van Veldhuisen DJ, van Woerden G, Gorter TM, van Empel VPM, Manintveld OC, Tieleman RG, et al. Ventricular tachyarrhythmia detection by implantable loop recording in patients with heart failure and preserved ejection fraction: the VIP-HF study. Eur J Heart Fail 2020; 22: 1923-9. [Crossref]

45. Bloch Thomsen PE, Jons C, Raatikainen MJ, Moerch Joergensen $\mathrm{R}$, Hartikainen J, Virtanen V, et al.; Cardiac Arrhythmias and Risk Stratification After Acute Myocardial Infarction (CARISMA) Study Group. Long-term recording of cardiac arrhythmias with an implantable cardiac monitor in patients with reduced ejection fraction after acute myocardial infarction: the Cardiac Arrhythmias and Risk Stratification After Acute Myocardial Infarction (CARISMA) study. Circulation 2010; 122: 1258-64. [Crossref]

46. Shen L, Jhund PS, Docherty KF, Petrie MC, Anand IS, Carson PE, et al. Prior Pacemaker Implantation and Clinical Outcomes in Patients With Heart Failure and Preserved Ejection Fraction. JACC Heart Fail 2019; 7: 418-27. [Crossref] 
47. Ong MK, Romano PS, Edgington $S$, Aronow HU, Auerbach $A D$, Black JT, et al.; Better Effectiveness After Transition-Heart Failure (BEAT-HF) Research Group. Effectiveness of Remote Patient Monitoring After Discharge of Hospitalized Patients With Heart Failure: The Better Effectiveness After Transition -- Heart Failure (BEATHF) Randomized Clinical Trial. JAMA Intern Med 2016; 176: 310-8. [Crossref]

48. Stehlik J, Schmalfuss C, Bozkurt B, Nativi-Nicolau J, Wohlfahrt P, Wegerich $S$, et al. Continuous Wearable Monitoring Analytics Predict Heart Failure Hospitalization: The LINK-HF Multicenter Study. Circ Heart Fail 2020; 13: e006513. [Crossref]

49. Barbosa RS, Glass L, Proietti R, Burstein B, Al-Turki A, Sobolik L, et al. Defining the pattern of initiation of monomorphic ventricular tachycardia using the beat-to-beat intervals recorded on implantable cardioverter defibrillators from the RAFT study: A computerbased algorithm. J Electrocardiol 2018; 51: 470-4. [Crossref]

50. Charitos El, Pürerfellner H, Glotzer TV, Ziegler PD. Clinical classifications of atrial fibrillation poorly reflect its temporal persistence: insights from 1,195 patients continuously monitored with implantable devices. J Am Coll Cardiol 2014; 63: 2840-8. [Crossref]

51. Andrade JG, Champagne J, Dubuc M, Deyell MW, Verma A, Macle $\mathrm{L}$, et al.; CIRCA-DOSE Study Investigators. Cryoballoon or Radiofrequency Ablation for Atrial Fibrillation Assessed by Continuous
Monitoring: A Randomized Clinical Trial. Circulation 2019; 140: 177988. [Crossref]

52. Nam Y, Kong Y, Reyes B, Reljin N, Chon KH. Monitoring of Heart and Breathing Rates Using Dual Cameras on a Smartphone. PLoS One 2016; 11: e0151013. [Crossref]

53. Laske TG, Iaizzo PA, Garshelis DL. Six Years in the Life of a Mother Bear - The Longest Continuous Heart Rate Recordings from a FreeRanging Mammal. Sci Rep 2017; 7: 40732. [Crossref]

54. Hannun AY, Rajpurkar P, Haghpanahi M, Tison GH, Bourn C, Turakhia MP, et al. Cardiologist-level arrhythmia detection and classification in ambulatory electrocardiograms using a deep neural network. Nat Med 2019; 25: 65-9. [Crossref]

55. Krahn AD, Klein GJ, Yee R, Skanes AC. Randomized assessment of syncope trial: conventional diagnostic testing versus a prolonged monitoring strategy. Circulation 2001; 104: 46-51. [Crossref]

56. Hoefman E, van Weert HC, Reitsma JB, Koster RW, Bindels PJ. Diagnostic yield of patient-activated loop recorders for detecting heart rhythm abnormalities in general practice: a randomised clinical trial. Fam Pract 2005; 22: 478-84. [Crossref]

57. Sivakumaran S, Krahn AD, Klein GJ, Finan J, Yee R, Renner S, et al. A prospective randomized comparison of loop recorders versus Holter monitors in patients with syncope or presyncope. Am J Med 2003; 115: 1-5. [Crossref] 